\title{
An unusual cause of hematospermia: Primary adenocarcinoma of the seminal vesicle
}

\author{
Alper Eken, MD; Volkan Izol, MD; ${ }^{*}$ I. Atilla Aridogan, MD* Seyda Erdogan, MD; Arbil Acıkalın, MD; \\ Zuhtu Tansug, MD*
}

*Cukurova University, Medical Faculty, Department of Urology, Adana, Turkey; 'Cukurova University, Medical Faculty, Department of Pathology, Adana, Turkey

Cite as: Can Urol Assoc J 2012;6(6):E259-E262. http://dx.doi.org/10.5489/cuaj.11132

\section{Abstract}

Adenocarcinoma of the seminal vesicles is one of the rare causes of hematospermia. Primary seminal vesicle adenocarcinoma is extremely rare and difficult to diagnose due to frequent invasion of adenocarcinomas of the surrounding organs, especially the prostate. In the present study, a case of a primary seminal vesicle adenocarcinoma will be discussed in the light of the current literature.

\section{Introduction}

Adenocarcinoma of the seminal vesicles is one of the rare causes of hematospermia. Primary seminal vesicle adenocarcinoma is extremely rare and difficult to diagnose due to frequent invasion of adenocarcinomas of the surrounding organs, especially the prostate. Since it was first defined, ${ }^{1}$ primary seminal vesicle adenocarcinoma has been reported in about 50 patients. In the present study, a case of a primary seminal vesicle adenocarcinoma will be discussed in the light of the current literature.

\section{Case report}

A 57-year-old male was admitted with urinary retention and 3 episodes of hematospermia within the last 6 months. At another centre, transurethral resection of the bladder plus a prostatic urethral biopsy had been performed one month before due to retention; at that time, the pathologic examination revealed high-grade papillary urothelial carcinoma and mixed adenocarcinoma.

During the visit at our centre, the rectal examination revealed a hard, palpable mass obstructing the lumen (grade 3/4). The prostate-specific antigen (PSA) level was $6.19 \mathrm{ng} /$ $\mathrm{mL}$. The serum carcinoembryonic antigen (CEA) and CA19-9 levels were normal; CA125 was $71.5 \mathrm{U} / \mathrm{mL}$ (range: 0-20 U/
$\mathrm{mL}$ ) and CA15-3 was $41.3 \mathrm{U} / \mathrm{mL}$ (range: $5-21 \mathrm{U} / \mathrm{mL}$ ). During the cystoscopy, there were no tumoural lesions; an indentation was observed from the interior and a mass lesion filling the seminal vesicle region was noted on bimanual examination. Paraffin blocks of the earlier operation were re-examined. Tumour cells with a hobnail appearance and transparent cytoplasm showing papillary and glandular structures were observed. Immunohistochemical evaluation demonstrated that CEA and cytokeratin 7 were positive, and PSA and cytokeratin 20 were negative. A magnetic resonance image showed: a $10 \times 15-\mathrm{cm}$ malignant lesion of cystic-necrotic nature with irregular solid components at the base and a widespread hematoma invading the prostate and the seminal vesicle with a clear-cut interphase between the tumour and the bladder (Fig. 1). According to results, the patient was diagnosed with a seminal vesicle adenocarcinoma.

A thin-walled cystic formation with an anterior surface of about $8 \mathrm{~cm}$ adhering to the bladder and sigmoid colon was noted on exploration. While the cystic formation was being excised from the surrounding tissues, due to perforation of the wall of the cystic formation, about $100 \mathrm{cc}$ of serous fluid was drained. Solid components at the base of the cystic formation were excised as much as possible; however, some residual mass strongly adherent to the bladder base and rectum had to be left behind. Pathologic examination revealed a malignant tumour exhibiting papillary and tubulopapillary structures with fields of transparent cells, solid regions and extracellular mucin deposition. Cytokeratin 7 was positive (Fig. 2), CEA was focally positive (Fig. 3), and cytokeratin 20 and PSA were negative by immunohistochemical methods.

On pelvic MRI evaluation during the first postoperative month, a $7 \times 5-\mathrm{cm}$ mass lesion was noted posterior to the bladder and adjacent to the prostate superiorly. A total dose of 50 Gy radiotherapy in 2 Gy fractions was applied between March 2006 and May 2006. An abdominopelvic computed tomography (CT) evaluation in July 2007 revealed several cystic lesions, the largest of which was $1.5 \mathrm{~cm}$ and adjacent 


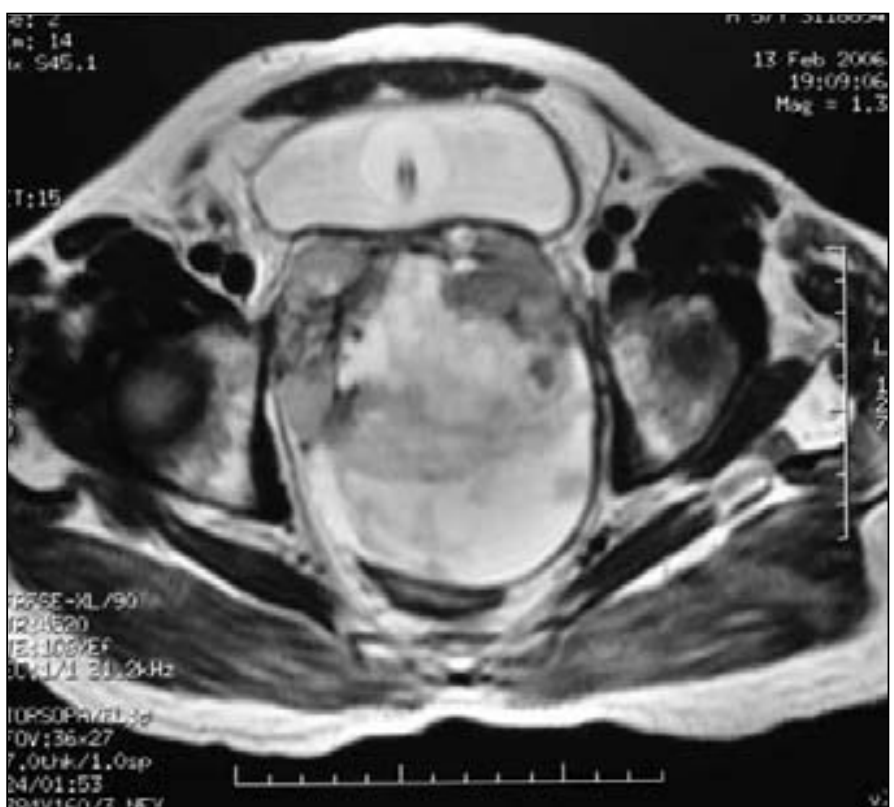

Fig. 1. The magnetic resonance image showing a $10 \times 15-\mathrm{cm}$ malignant lesion.

to the prostate on pelvic sections. The PSA level was $1 \mathrm{ng} /$ $\mathrm{mL}$. Positron emission tomography (PET) performed about 6 months later revealed F-18 FDG involvement in multiple lymph nodes in the left cervical chain (maximum $1.5 \mathrm{~cm}$ ), two nodular pulmonary lesions (maximum $2 \times 2.3 \mathrm{~cm}$ ); an infrarenal para-aortic lymph node $(1.5 \times 2.5 \mathrm{~cm})$; and a $4 \times 2.5 \mathrm{~cm}$ mass between the bladder the right distal ureter. Based on the PET result, $50 \mathrm{mg} / \mathrm{m}^{2}$ of cisplatin (days 1-2) and $1000 \mathrm{mg} / \mathrm{m}^{2}$ of 5 -FU (days $1-4$ ) were initiated. The patient had his sixth chemotherapy course in August 2007 and did not come back for more visits. When the patient's family was called, we were informed that the patient had died in February 2008 due to a nosocomial pulmonary infection at another centre.

\section{Discussion}

Infectious conditions are the most common etiologic factor for hematospermia accounting for about $40 \%$ of all cases. Inflammatory conditions, neoplasms, iatrogenic factors and systemic diseases are other etiologic factors for hematospermia (Table 1). ${ }^{2,3}$ Prolonged sexual abstinence, excessive masturbation and rigorous sexual intercourse should also be considered as causes of hematospermia. ${ }^{3}$ After confirming actual hematospermia, physicians should perform a comprehensive evaluation, including history and physical exam. Although there are no specific guidelines for hematospermia, the American (AUA) and Canadian Urological Association (CUA) guidelines include hematospermia in prostate infection and the use of transrectal ultrasound in the etiology of hematospermia. ${ }^{4,5}$ Genital organs should be evaluated on physical and rectal exam to rule out prostate cancer. During

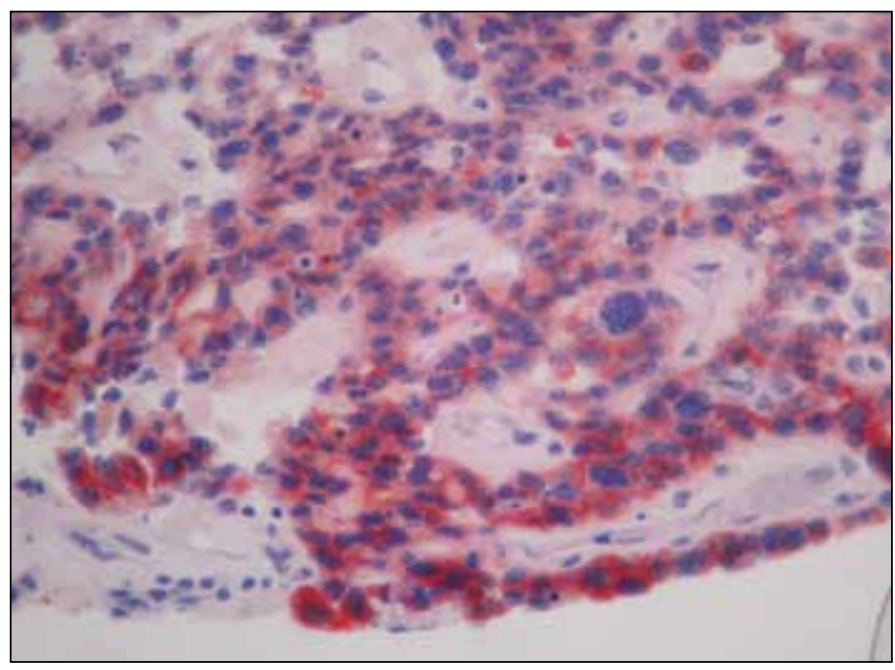

Fig. 2. The pathologic examination revealed a malignant tumor exhibiting papillary and tubulopapillary structures with fields of transparent cells, solid regions, and extracellular mucin deposition. The cytokeratin 7 was positive.

the rectal exam, if seminal vesicles are palpable, an underlying pathology must be excluded. Laboratory investigations for hematospermia include urine analysis and culture, PSA, semen analysis and urine cytology. If a sterile pyuria is noted, tuberculosis should be ruled out. If sexually transmitted diseases are suspected, urethral cultures to confirm gonorrhea and chlamydia should be made. Transrectal ultrasonography is recommended as the first imaging method since it allows for a simultaneous biopsy and has a benefit of diagnosing

\section{Table 1. Etiologic factors for hematospermia}

Inflammatory conditions

Neoplasms

latrogenic factors

Systemic diseases
Epididymitis neoplasms (prostate, seminal vesicle and testicular cancer, melanoma

Cystitis

Prostatitis

Seminal vesiculitis

Condylomata of urethra and meatus

Cytomegalovirus

Schistosmiasis

Schistosmiasis

Hydatid

Prostate

Seminal vesicle and testicular cancer

\section{Melanoma}

Prostate biopsy

Prostatic surgery

Transurethral instrumentation

Self-urethral instrumentation

Radiotherapy

Amiloidosis

Bleeding disorders

Chronic liver disease 


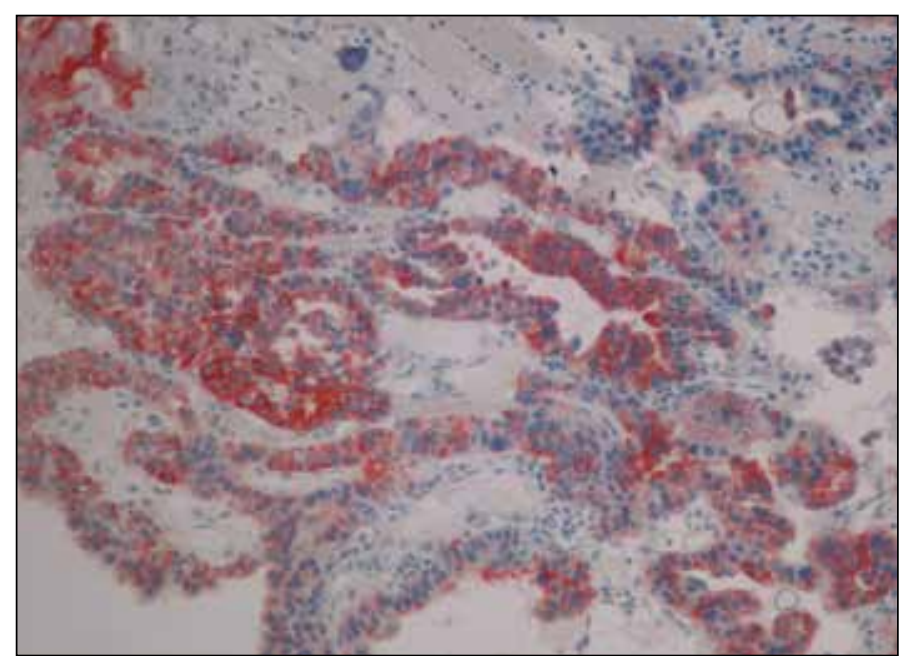

Fig. 3. The carcinoembryonic antigen was focally positive.

calculi, cysts, Mullerian duct remnants, prostatic varices and inflammatory changes. ${ }^{3}$ Individuals who are middleaged with recurrent hematospermia need special attention and further evaluation. In cases of persistent hematospermia, a detailed radiological assessment may ascertain the exact cause. In these patients, neoplastic diseases should be excluded. They also should be followed up for a limited time. ${ }^{3}$ It should always be kept in mind that although hematospermia is rare in a prostate cancer screening population, it is associated with an increased risk of subsequent prostate cancer detection. ${ }^{6}$

Although seminal vesicle adenocarcinoma can be observed in males at every age, the mean patient age at the time of diagnosis is 62 years (range: $13-90$ years). ${ }^{7,8}$ The most frequent symptoms in seminal vesicle adenocarcinomas are bladder outlet obstruction and hematospermia. Nearly 35\% of patients also complain of dysuria. ${ }^{9}$ Even though palpation of solid tumour tissue on rectal exam is one of the essential features in diagnosis, in recent studies no abnormal findings have been detected in $30 \%$ of the patients on rectal examination. ${ }^{7,8,9}$ Cystoscopic evaluation is normal in $20 \%$ of patients. ${ }^{8-10}$ Radiologic evaluations performed in the early stage of disease may exclude carcinomas of neighbouring organs. Prostate neoplasms should be excluded by PSA and rectal exam in all cases. Sigmoidoscopy should also be performed to exclude cancer of the rectum.

The diagnostic criteria for seminal vesicle adenocarcinoma were modified by Benson. ${ }^{7}$ The seminal vesicle should be verified macroscopically and microscopically to confirm a carcinoma. Moreover, to confirm that the tumour is completely or essentially localized to the seminal vesicles, the PSA, PAP, and CEA should be negative. It can be differentiated from PSA (negative) and prostatic acid phosphatase (PAP)-positive prostate cancer and CEA-positive rectum cancer. Less differentiated prostate carcinomas may be PSA (negative) and PAP (negative); there should not be a primary carcinoma focus in any other body region. ${ }^{8-15}$

Normal serum levels of PSA and CEA prevent invasion of the prostate or colon carcinoma. However, increased serum CEA levels can also be observed in rare cases of seminal vesicle adenocarcinoma. CA-125 and cytokeratins 7 and 20 provide more valuable information for differentiating seminal vesicle adenocarcinoma from invasion of other organs by tumours (Table 2$).^{8-15}$

The prognosis of seminal vesicle adenocarcinoma is often poor due to delayed diagnosis. About $95 \%$ of the patients die in less than 3 years. ${ }^{711,13}$ The primary goal of treatment is surgical resection of the tumour. Ejaculatory canals are frequently invaded; therefore, prostatectomy should also be performed. ${ }^{9}$ Radiotherapy can be applied as another treatment modality in the presence of postoperative positive surgical borders or residual tumour. However, radiotherapy is not an effective treatment. ${ }^{9,10}$ There has been no definitive recommendation regarding the efficacy of chemotherapy and the chemotherapy regimen that should be used in primary seminal vesicle adenocarcinomas. ${ }^{7}$ In our case, despite the use of every treatment method, the time from admission to death was 24 months.

\section{Conclusion}

In patients admitted with hematospermia, malignancy should be considered as well as non-specific infections. Low-volume, single episode hematospermia is often self-limiting and observation is the most appropriate management. However, men with persistent hematospermia and associ-

$\begin{aligned} & \text { Table 2. Immunohistochemical findings in neighboring organ carcinomas which may cause primary seminal vesicle } \\
& \text { adenocarcinoma and seminal vesicle invasion }\end{aligned}$
\begin{tabular}{lccccc} 
Immunohistochemical test & $\begin{array}{c}\text { Seminal vesicle } \\
\text { adenocarcinoma }\end{array}$ & Prostate cancer & Bladder cancer & Rectum cancer & $\begin{array}{c}\text { Adenocarcinoma of the } \\
\text { Mullerian duct }\end{array}$ \\
\hline CA 125 & + & - & - & - \\
Cytokeratin 7 & + & $+/-$ & $+/-$ & - \\
PSA & - & + & - & - \\
Cytokeratin 20 & - & $+/-$ & $+/-$ & + \\
CEA & $+/-$ & - & - & - \\
\hline
\end{tabular}

PSA: prostate-specific antigen; CEA: carcinoembryonic antigen. It should be kept in mind that variations may be observed in poorly differentiated or anaplastic tumours. 
Eken et al.

ated symptoms need more extensive evaluation. Although prostate cancer is the most frequently considered diagnosis among the neoplastic diseases, primary tumours of the seminal vesicles or neighbouring organ tumours causing seminal vesicle invasion should also be kept in mind.

Competıng interests: None declared.

This paper has been peer-reviewed.

\section{References}

1. Lyons 0. Primary carcinoma of the left seminal vesicle. J Urol 1925;13:477.

2. Stefanovic KB, Gregg PC, Soung M. Evaluation and treatment of hematospermia. Am Fam Physician 2009:80:1421-7.

3. Kumar P, Kapoor S, Nargund V. Hematospermia - a systematic review. Ann R Coll Surg Engl 2006;88:33942. http://dx.doi.org/10.1308/003588406X1 14749

4. http://www.auanet.org/content/guidelines-and-quality-care/policystatements/c/consensus-statementon-urologic-ultrasound-utilization.ffm
5. Nickel JC. Prostatitis. Can Urol Assoc J 2011;5:306-15. http://dx.doi.org/10.5489/cuaj.11211

6. Han M, Brannigan RE, Antenor JAV, et al. Association of hemospermia with prostate cancer. J Urol 2004;172:2189-92. http://dx.doi.org/10.1097/01.ju.0000144565.76243.bl

7. Benson RC, Clark WR, Farrow GM. Carcinoma of the seminal vesicle. J Urol 1984;132:483-5.

8. Kawahara $M$, Matsuhashi $M$, Tamima $M$, et al. Primary carcinoma of seminal vesicle. Diagnosis assisted by sonograpy. Urology 1988;32:269. http://dx.doi.org/10.1016/0090-4295(88)90399-8

9. Thiel R, Effert P. Primary adenocarcinoma of the seminal vesicles. J Urol 2002;168:1891-6. http:// dx.doi.org/10.1016/S0022-5347(05)64260-7

10. Davis NS, Merguerian PA, DiMarco PL, et al. Primary adenocarcinoma of the seminal veside presenting as bladder tumor. Urology 1988;32:466. http://dx.doi.org/10.1016/0090-4295(88)90429-3

11. Chhabra A, Bhullar SS, Oberoi R, et al. Primary adenocarcinoma of seminal vesicles. Ind I Radiol Imag 2002; $12: 75-7$.

12. Ohmori T, Okada K, Tabei R, et al. CA 125-producing adenocarcinoma of seminal vesicle. Pathol Int 1994;44:333-7. http://dx.doi.org/10.1111/i.1440-1827.1994.tb03372.x

13. Ormsby AH, Haskell $R$, Jones $D$, et al. Primary seminal vesicle carcinoma: an immunohistochemical analysis of four cases. Mod Pathol 2000; 13:46-51. http://dx.doi.org/10.1038/modpathol.3880008

14. Moll R. Cytokeratins in the histological diagnosis of malignant tumors. Int I Biol Marker 1994;9:63-9.

15. Tarian M, Ottlez I, Tot T. Primary adenocarcinoma of the seminal vesicle. Ind J Urol 2009;25:143-5. http://dx.doi.org/10.4103/0970-1591.45557

Correspondence: Dr. I. Atilla Aridogan, University of Cukurova Faculty of Medicine, Department of Urology, 01330 Adana, Turkey; fax: +90 322 4573072; aridogan@cu.edu.tr 